Promoción de hábitos saludables jugando con el niño en el ámbito familiar: la oca saludable

\title{
Encouraging healthy habits by playing with the child in the family milieu: the snakes and ladders game
}

\author{
M. Sánchez Echenique', R. Pèlach ${ }^{2}$
}

\section{RESUMEN}

La sociedad está sufriendo muchos cambios en las últimas décadas y estos han incidido fuertemente en la morbilidad y la mortalidad infantil. Enfermedades prácticamente desaparecidas por el impacto de las vacunaciones y la mejora del nivel de vida han dejado paso a otras derivadas de los cambios de la alimentación y estilo de vida; los accidentes, por otra parte, han pasado a ser la primera causa de morbilidad y mortalidad una vez pasado el primer año de vida.

Las estrategias deben ir encaminadas a evitar las causas de esta nueva manera de enfermar.

Se presenta un material lúdico, "La oca saludable", que promueve unos hábitos sanos de vida mediante el juego de la oca. Con el fin de que el niño vaya interiorizando, mediante el juego, unos hábitos seguros, se han sustituido determinadas casillas de éste por unas viñetas positivas, en las que el niño realiza actividades saludables, siendo premiado por ello, y otras en las que realiza actividades de riesgo, siendo penalizado. Las áreas tratadas son, hábitos (nutrición, sueño, estudio, juegos, etc.), accidentes caseros, seguridad vial (peatón, ciclista y viajero de automóvil), y piscinas. El juego comienza con un niño de 3-4 años y la meta se alcanza como un adolescente sano.

Dado que la variación de personalidades es enorme, creemos que el complemento a las medidas legislativas, educativas y campañas generales en los medios de comunicación debe ser el medio familiar, el que más conoce al niño y donde éste aprende sus más profundas actitudes. Este juego puede ser un apoyo para los padres en esta difícil tarea.

Palabras clave. Oca saludable. Prevención. Hábitos. Accidentes. Infancia.

\begin{abstract}
In recent decades society has been undergoing many changes and these have had a strong incidence on infant morbidity and mortality. Diseases that have practically vanished due to the impact of vaccinations and the improvement of the standard of living have given way to others derived from the changes in eating habits and life style; accidents, on the other hand, have come to be the first cause of morbidity and mortality after the first year of life has been passed.
\end{abstract}

Strategies must be directed towards avoiding the causes of this new way of falling ill.

We present a Spanish version of a game similar to "snakes and ladders" called "the healthy goose", which encourages healthy living habits. In order that the child should internalize safe habits by means of the game, certain squares in the game have been replaced by positive drawings, in which the child carries out healthy activities, receiving prizes for these, and others in which risky activities are performed, which are penalized. The areas dealt with are: habits (nutrition, sleep, study, games, etc.), Accidents at home, road safety (pedestrian, cyclist and automobile traveler), and swimming pools. The game starts with a child of 3-4 years and the goal is reached as a healthy adolescent.

Given that the variation in personalities is enormous, we believe the family milieu should be the complement to legislative and educational measures and general campaigns in the means of communication, since it is what the child knows best and where he learns his deepest attitudes. This game could be a help to parents in this difficult task.

Key words. Healthy goose. Prevention. Habits. Accidents. Childhood.

ANALES Sis San Navarra 2002; 25 (Supl. 2): 85-92.

1. Pediatra. Unidad de Programación y Docencia. Dirección Atención Primaria. Servicio Navarro de Salud.

2. Pediatra. Atención Primaria. Centro de Salud de Barañáin. Servicio Navarro de Salud.

\section{Correspondencia:}

Manuela Sánchez Echenique

Unidad de Programación y Docencia

Plaza de la Paz, s/n - 6 $6^{\mathrm{a}}$ planta

31002 Pamplona (Navarra)

Tfno. 948429351

Fax 948429384

E-mail: msanchee@cfnavarra.es 


\section{INTRODUCCIÓN}

La morbi-mortalidad infantil durante las pasadas décadas han sufrido un gran cambio en todos los países industrializados.

La disminución de las enfermedades transmisibles y la mejora de la calidad de vida en el mundo occidental ha originado la casi desaparición de las enfermedades históricamente conocidas como enfermedades propias de la infancia, paralelamente a la aparición de otras relacionadas con los cambios en los hábitos.

Estos cambios de hábitos tienen su punto culminante en la época de la adolescencia, donde estamos asistiendo al incremento de lesiones traumáticas, embarazos no deseados, abuso de drogas y alcohol y enfermedades de transmisión sexual, incluyendo el HIV y desórdenes alimenticios.

Todos estos datos han hecho plantearse la urgente necesidad de programas educacionales y sanitarios que aborden los nuevos problemas que, en ocasiones, tienen carácter epidémico.

La AMA (American Medical Association), en sus GAPS (Guidelines for Adolescent Preventive Services) recomienda que los proveedores de servicios deben de tener un componente mayor en su área clínica.

Analizando las causas más frecuentes de morbilidad y mortalidad en niños mayores de 1 año, se aprecia que los accidentes ocupan un papel principal por lo que, dejando aparte las enfermedades agudas, parece oportuno dedicar también nuestros recursos a disminuir las causas que los originan.

La palabra accidente, del latín, accidens-accidentis, tiene empleo en muchas situaciones, pero, en general, se aplica al "suceso eventual que altera el orden regular de las cosas"; a su vez, el suceso eventual (adjetivo derivado de evento del latín eventus) significa hecho imprevisto. Hay un sentido de azar, de no comprensible y en consecuencia no previsible, por lo que en muchas situaciones la utilización de esta palabra para referirse a sucesos, que se podían haber evitado con ciertas medi- das, crea la idea de que hay que resignarse. Sería pues mejor evitar esta palabra y hablar de lesiones inintencionadas. Estudios hechos por Peterson ${ }^{1}$ acerca de los pensamientos de los padres apreciaron que la variable más asociada se daba entre los padres que, utilizando métodos de seguridad y empleando tiempo en enseñar a sus hijos habilidades para autoprotegerse, habían manifestado que creían que las medidas de enseñanza de habilidades seguras podrían prevenir lesiones en sus hijos. Esto iría a apoyar la idea de que hay una correspondencia entre el significado de la palabra y la actitud que se toma ante esta problemática, por lo que habrá que tenerlo en cuenta a la hora de diseñar intervenciones.

Parece haber unanimidad en la importancia de la promoción de hábitos saludables y un entorno adecuado en los primeros años de la vida para que el niño llegue a la adolescencia sano y con una autonomía física y mental que le permita cuidarse a sí mismo adecuadamente.

Aunque hay muchos tipos de intervención se podrían dividir en tres grandes grupos:

1. Campañas de Salud Pública: folletos, anuncios en los medios, pósters advirtiendo de los riesgos o recomendando medidas. Estudios controlados no han demostrado su efectividad a largo plazo como única medida de intervención

2. Legislativas: identificación sistemática y reducción de causas del entorno para prevención de accidentes. Es la medida más efectiva.

3. Consejo individual: varios estudios en las pasadas décadas han indicado que los profesionales sanitarios pueden jugar un papel primordial en la prevención de lesiones a través de los consejos anticipativos a los padres y al niño (según edad) sobre medidas de seguridad.

Además de las medidas destinadas a la población en general las dirigidas al propio niño en desarrollo deben de incidir en la adquisición de habilidades saludables que le capaciten en la utilización adecuada de 
los juegos, así como un orden en el resto de actividades cotidianas.

Muchas de las patologías de las naciones desarrolladas están presentando unas características de epidemia debido al número de personas afectadas, con el coste en los tratamientos que ello supone, convirtiéndose en un problema de salud pública, bien en este momento o tras un periodo de tiempo.

Identificar los factores y establecer programas para erradicarlos o disminuirlos debe pues ser una de las principales estrategias.

Existen diversas formas de abordar la promoción de la salud directamente con el niño. Ya en la escuela, a través de las asignaturas "transversales", recibe conocimientos acerca del funcionamiento de su cuerpo, de los tipos de alimentos y su composición, así como otras informaciones acerca de otros agentes (drogas, etc.) que inciden en la salud; como un complemento a estos materiales y actividades presentamos una propuesta didáctica para los niños.

Se trata de la "Oca saludable", un juego que pretende promocionar los hábitos saludables en los niños, preferentemente jugando en familia.

\section{LA "OCA SALUDABLE"}

Los niños no tienen desarrolladas las capacidades de comprender la repercusión que pueden tener en su salud las acciones que realizan, sobre todo en el largo plazo. Tienen vivencias cambiables sobre su propio cuerpo y a lo largo del desarrollo van disminuyendo su dependencia del sistema familiar y aumentando su autonomía. Resulta improcedente pues, pretender que hagan o dejen de hacer algo con el argumento de que cuando sean adultos de 40-50 años pueden tener problemas si mantienen unos hábitos de vida.

Por este motivo, es importante encontrar procedimientos que incentiven estilos de vida saludables, tanto individuales como de grupo. Estas actuaciones tenderían a promover hábitos con efecto a corto y largo plazo, bien para prevenir alteraciones de nutrición o lesiones y muertes evitables en la infancia, como para la preven- ción de los factores de riesgo ligados a las principales causas de muerte en la vida adulta.

Como otra aportación a sumar al material que los niños ya tienen en la escuela, ideamos otra manera de abordar este tema mediante el juego. El juego es una característica esencial de la niñez, común a los niños de todas las latitudes. De hecho, un niño que no juegue, teniendo la posibilidad de ello, es muy probable que tenga algún problema que hay que investigar.

Una vez decidido efectuar un material de este tipo, se escogió el juego de la oca, porque es conocido por la mayoría de nosotros y puede servir incluso para que juegue toda la familia, lo que favorecería que se comenten los temas sin necesidad de personalizar; a veces, al niño le toca aceptar lo que le dicen los mayores y cuando no cumple se siente culpable. Ver como protagonista a un tercero permite al niño verse representado sin necesidad de recibir una reprimenda e ir interiorizando conceptos de lo que es positivo o negativo para su salud.

Como en el juego tradicional, hay casillas neutras, positivas y negativas.

Las positivas, en las que se premian conductas o hábitos saludables, recalcan el aspecto positivo de la acción y permiten que se avance más rápido en el juego.

En las negativas, se penalizan hábitos que pueden ser perjudiciales a largo plazo o acciones que pueden producir lesiones, incluso mortales. Se detiene el avance e incluso se hace retroceder casillas.

Las casillas van pasando por la etapa preescolar (3-5 años, 20 casillas), escolar (6-9 años, 20-40 casilla) y pre-adolescente (10-12 años, 40-60 casilla).

La meta es llegar a un adolescente sano.

\section{ELABORACIÓN}

En la elaboración se ha dado al dibujante una idea para el diseño de la viñeta y se ha puesto un texto relacionado con ella, intentando dar un tono lúdico, e incluso rimado a la acción con su efecto. 
En cuanto a los temas tocados, por un lado se han escogido los hábitos que se sabe tienen repercusión en la edad adulta y por otro los relacionados con la causa más frecuente de morbilidad y mortalidad en la infancia de los países desarrollados, los accidentes. Expondremos más adelante los datos que nos han hecho incidir tanto en estos, fundamentalmente los ocurridos fuera del domicilio. Así, tenemos casillas que tratan:

- Hábitos de vida:

- Alimentación, sueño, ocio, estudio, etc., incidiendo en los que, para los niños, presentan mayor dificultad de cumplimiento (comer verduras, frutas, limpiarse los dientes, beber leche, pocas chucherías, dormir suficiente, tiempo para jugar, estudiar y leer).

- Accidentes caseros.

- Fuegos y cerillas.

- Caídas desde altura ( Hay niños que creen que se puede volar).

- Ingesta de medicamentos o sustancias.

- Seguridad vial.

- Como peatón.

- Como ciclista, insistiendo en el uso del casco.

- Cinturones y asientos adecuados en el automóvil.

- Piscinas.

- Quemaduras solares (prevención de quemaduras y cáncer de piel).

- Saltos de cabeza (prevención de lesiones medulares).

- No aceptar propuestas de desconocidos.

- No fumar cigarrillos.

De estos temas, hay viñetas en las que el protagonista se comporta de una manera positiva y por lo tanto es premiado; en caso contrario, a mayor peligro, mayor penalización (Fig. 1).

Se promueve también el juego limpio y sin peleas. Por último, el simple hecho de jugar en familia y poder comentar casillas en conjunto puede tener aspectos positivos en la interiorización de estos conceptos.
También añadir que, durante el juego, cuando el niño cae en una casilla penalizada y debe de retroceder, además de ir comprendiendo el motivo, aprende asimismo que hay veces que se pierde y hay que volver hacia delante, como en las dificultades de la vida real. A los niños no les gusta perder, a veces lo hacen de una manera explosiva, lloran, tienen accesos de rabia, según edades, pero deben ir aprendiendo durante la infancia a no obtener las cosas de una manera inmediata, para ir aumentando su capacidad de frustración; el jugar puede ayudarles a madurar también este aspecto.

\section{DISCUSIÓN}

Se ha hablado mucho del impacto que tienen las informaciones; durante la presentación del juego con motivo de la feria de muestras, pudimos apreciar en el stand donde se instaló una oca saludable gigante, que los grupos de niños que acudieron con sus maestros, conocían bastante bien los alimentos, etc. y su importancia, la indicación de usar cinturón y casco; sin embargo, pocos lo tenían y de estos una mínima parte lo utilizaba, lo que da una idea del campo que hay que trabajar para conseguir interiorizar la información. $\mathrm{Al}$ acabar, parecía que habían descubierto el significado real de lo que les habían enseñado en la escuela.

El motivo de dedicar un número considerable de casillas a mejorar las habilidades seguras se muestra seguidamente:

Grosman $^{2}$ en Seattle, ha comprobado que las causas de la mitad de los accidentes que atienden son debidas a caídas de bici, ahogamientos en piscinas y lesiones a peatones, causas todas que son evitables en un gran porcentaje.

Por otro lado, Chiolero $^{3}$ en Lausanne ha verificado que el $45 \%$ de los escolares entre 10-14 años había sido visto en el año anterior por una enfermera o médico por haber sufrido lesiones y Suiza tiene una tasa de 793/1.000 adolescentes por año lesionados. También entre estos el 10\% ha tenido 3 ó más lesiones por año.

Kingma ${ }^{4}$ en Groningen, entre escolares de 4 a 13 años vio que iba variando el numero de lesiones; pasaban del 0,5\% a 
los 4-5 años al 4,8\%o a los 10-12 años; el $59 \%$ eran en las extremidades superiores, predominantemente en lesiones de la muñeca, y el $33 \%$ en las extremidades inferiores, con lesión en el pie en la mitad de los casos y un $36 \%$ en el tobillo. En cuanto a las circunstancias, eran caídas en el 53\% de los casos (60\% en el propio suelo y el $35 \%$ haciendo ejercicio en barras, etc.).

King MA $^{5}$ estudió en Ontario, Canadá, los patrones de conducta entre la juventud canadiense; pero, aunque el $36 \%$ de los jóvenes refería haber tenido alguna lesión en los 12 meses anteriores, no encuentra evidencias que los de mayor alto riesgo tengan hábitos adversos, por lo que, ante las cifras existentes, propone llevar un seguimiento para estudiar las causas.

Todos han evidenciado un aumento de las lesiones según van avanzando los años, lo que demuestra que, a pesar de que el niño va aumentando en capacidad de razonamiento, ello no es suficiente ya que parece hallar otros intereses que le impiden pensar en las consecuencias, a veces dramáticas y cuando menos, penosas de algunas de las actividades que realiza sin la suficiente protección o habilidad. Los juegos que parecen tener más implicación en lesiones son los de balón.

Rosembeg $^{6}$, en Atlanta, propone incluir en este tipo de lesiones también las intencionales, puesto que si no se contempla como un problema de salud pública, estudiando sistemáticamente las causas, se pierde la oportunidad de prevenir este tipo de sucesos, afortunadamente mucho menos frecuentes en nuestro país que en EE.UU. En ocasiones, peleas que acaban en lesiones son consideradas "accidentes", y por lo tanto fortuitas, cuando tienen como razón última una incapacidad de resolver las discrepancias hablando o de asumir deportivamente una derrota; cuando este problema lo presenta un número elevado de personas, jóvenes en este caso, debería ser considerado un problema que puede tener resultados completamente diferentes según sea abordado de una manera u otra.

Stefansdottir ${ }^{7}$, en Reykjavik, ha visto que las lesiones tras campañas han descendido algo en los primeros años de vida, pero han aumentado levemente en la adolescencia. Aunque la mortalidad ha disminuido en general, tienen que seguir intentando disminuir las tasas de lesiones.

Por último, destacar a Navascués del Río y $\mathrm{col}^{8}$, en Madrid, que ha creado el primer registro español de traumas, con el fin de conocer la epidemiología y por lo tanto tener datos para desarrollar campañas preventivas posteriores.

En cuanto a las lesiones en piscinas, una de las áreas que más hemos insistido es la relativa a la precaución de las zambullidas, ya que el año 1998, según datos del Hospital de Parapléjicos, se atendió 10 pacientes con lesión medular por zambullida, siendo esta la causante del $6 \%$ de las lesiones medulares.

Pensando en el presente y futuro de los niños, habrá que establecer estrategias para que lleguen con hábitos saludables, que les permitan llegar a una vida adulta satisfactoria, pero además que, durante la edad de su desarrollo, adquieran las habilidades suficientes para disfrutar de un ocio que no les lesione y limite la vida, a veces para siempre. El auge de actividades deportivas cada vez más excitantes y arriesgadas, está originando que los jóvenes más dotados sean los más lesionados, ya que sus pares menos capacitados no se arriesgan tanto. Otro ejemplo significativo lo forman los parques de atracciones, en los que las personas hacen cola para subirse a ingenios mecánicos que tienen giros, rotaciones, descensos bruscos, "puenting", etc., cada vez más bruscos, como una muestra de la necesidad de buscar nuevas sensaciones.

Mostrar y participar con los niños en juegos, deportes y actividades lúdicas que puedan ejercitarse por un abanico amplio de edades y condiciones físicas puede permitir, por un lado reducir lesiones y, por otro, sentar las bases para una afición que permitirá disfrutarla en la vida adulta, incluso cuando la edad o condición física no sean adecuadas para otras. Asimismo, enseñar a disfrutar de actividades que se practican aisladamente (lectura, música, etc.) puede ser fundamental para evitar el aburrimiento cuando la persona, el niño en este caso, no tiene un grupo para compar- 
tir el ocio; el ejemplo es fundamental en estas edades. En esta era de las telecomunicaciones parece difícil conseguir que un niño lea un libro completo, pero es imprescindible abrir una ventana al mundo imaginario de todas las historias escritas por tantos autores a lo largo de la historia.

Este nuevo material fue editado por un convenio entre el Departamento de Salud y el diario local de mayor tirada que financió la edición y distribución con la prensa diaria. Por último, deseamos un futuro feliz a nuestros jóvenes y esperamos haber aportado un material que les haga disfrutar, mientras interiorizan hábitos que les ayudarán a mantenerse sanos.

Agradecimientos

Al Departamento de Salud que apoyó la propuesta y al Diario de Navarra que financió su edición y distribución; se distribuyeron 60.000 ejemplares a través de la prensa diaria y 20.000 a través de los centros de salud y colegios. Asimismo, fue presentada en la feria de muestras, en una edición gigante, que fue visitada por los escolares acompañados por sus profesores, pudiendo apreciarse una buena acogida del juego por parte de los niños.

\section{BIBLIOGRAFÍA}

1. Peterson L, Farmer J, Kashani JH. Parental injury prevention endeavors: a function of health beliefs? Health Psychol 1990; 9: 177191.

2. GRossman DC. The history of injury control and the epidemology of child and adolescent injuries. Future Child 2000 Spring-Summer; 10: 23-52.

3. Chiolero A, Schmid H. Accidental morbidity in adolescence: a retrospective study in 12 to 15 year-old school children in Switzerland. Schweiz Med Wochenschr 2000; 16: 130.

4. Kingma J, Ten Duis HJ. Injuries due to school sports accidents in 4 to 13 year-old children. Percept Mot Skills 2000; 90: 319-325.

5. King MA, Pickett W, KIng AJ. Injury in Canadian youth: a secundary analysis of the 1993-94 health behaviour in school-aged children survey. Can J Public Health 1998; 89: 397-40.

6. Rosenberg ML, Rodríguez JG, Ghorba TL. Childhood injuries: where we are. Pediatrics 1990; 86: 1084-1091.

7. Stefandottir A, Mogensen B. Epidemiology of childhood injuries in Reykjavik 1974-1991. Scand J Prim Health Care 1997; 15: 30-34.

8. NAVAsCuÉs del Río JA, Romero-Ruiz RM, SOlEToMartín J, CERDA-BenOCAL J, BarrientosFERnÁNDEZ G, SÁNCHEZ-MARTín R et al. First Spanish trauma registry: analysis of 1,500 cases. Eur J Pediatr Surg 2000; 10: 310-318. 


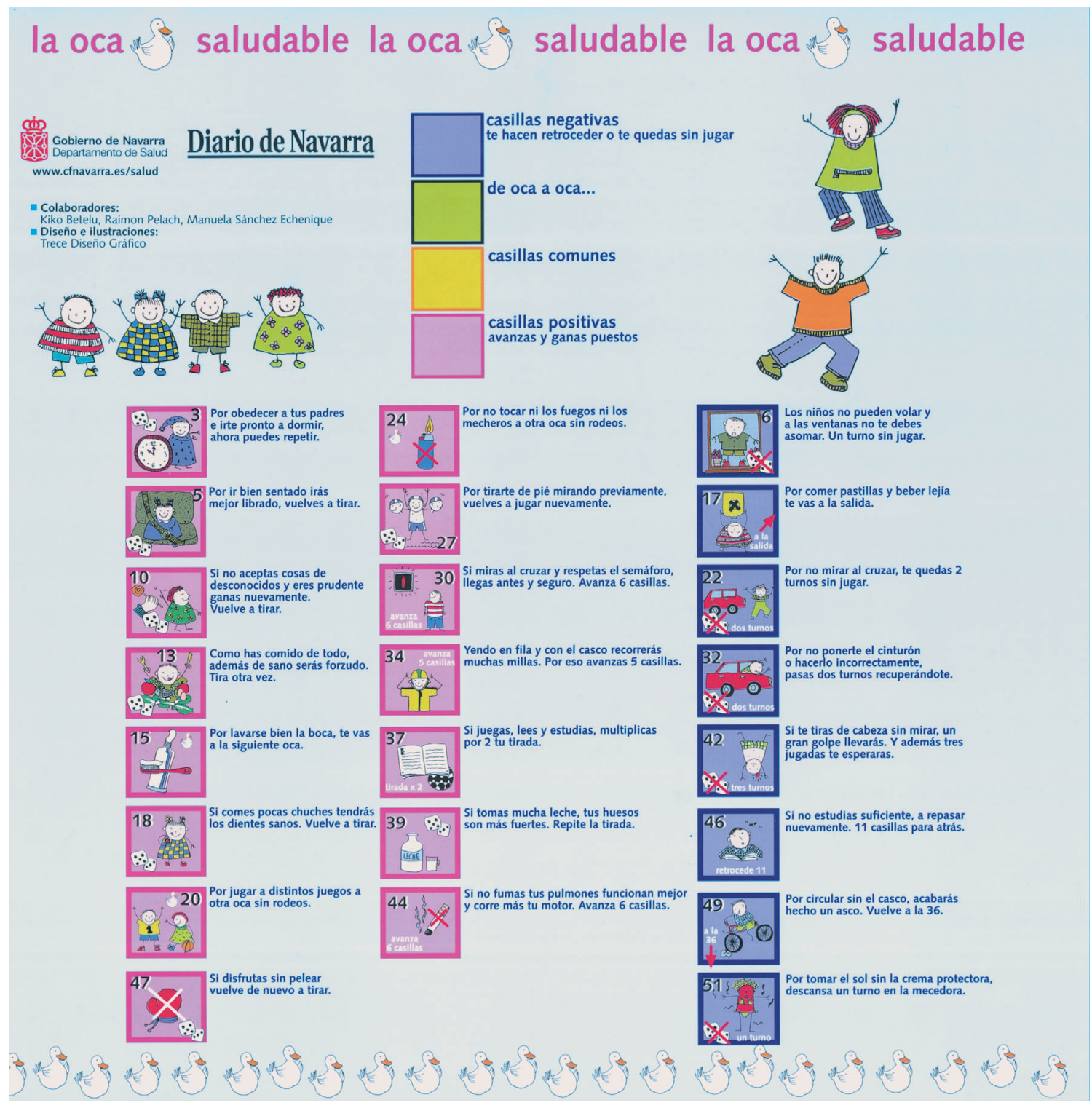




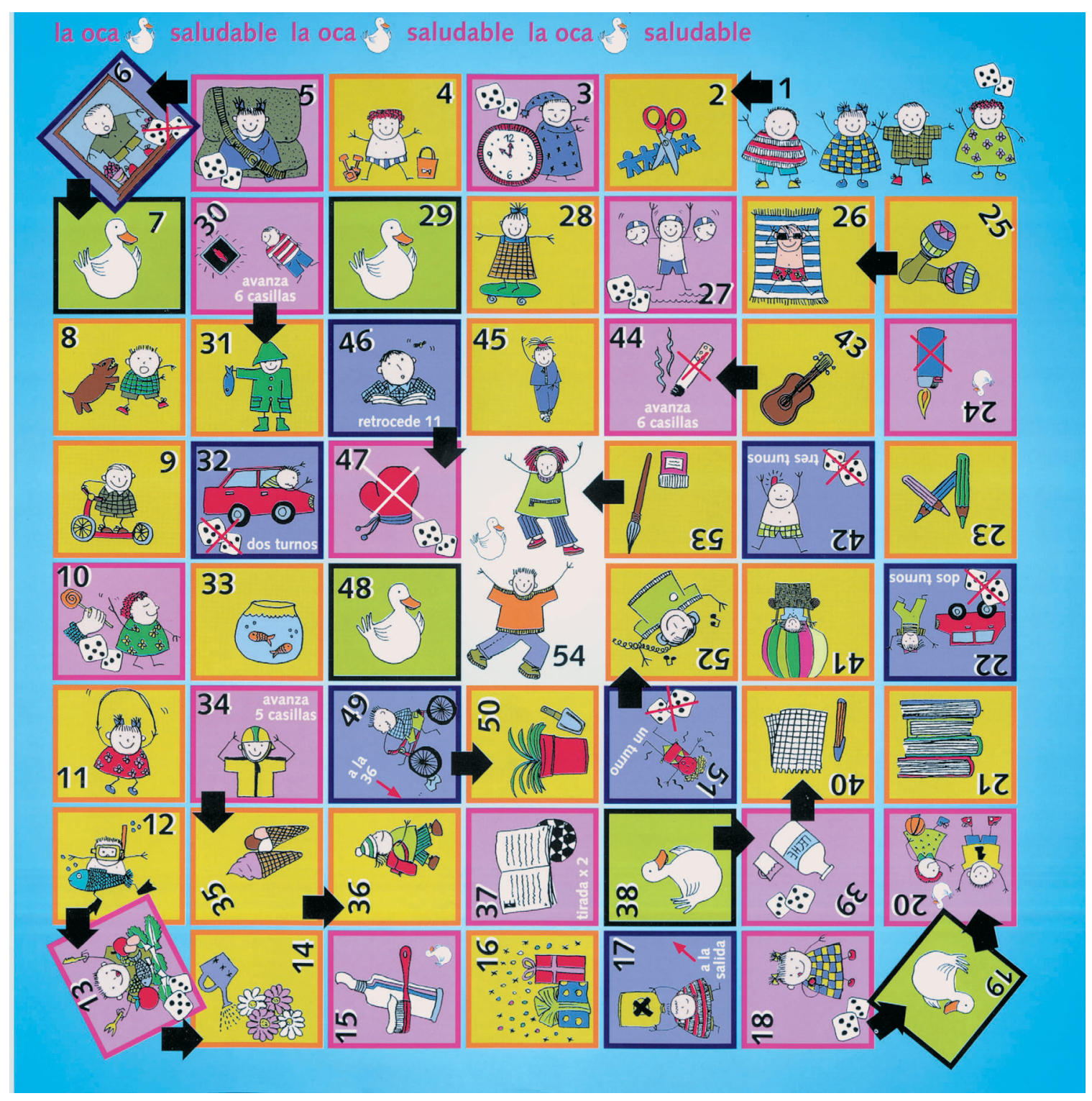

